\title{
Utility of Approximate Entropy from Overnight Pulse Oximetry Data in the Diagnosis of the
} Obstructive Sleep Apnea Syndrome

Roberto Hornero ${ }^{1}$, Member, IEEE, Daniel Álvarez ${ }^{1}$, Daniel Abásolo ${ }^{1}$, Member, IEEE, Félix del Campo $^{2}$, and Carlos Zamarrón ${ }^{3}$

${ }^{1}$ E.T.S. Ingenieros de Telecomunicación, University of Valladolid, Spain

${ }^{2}$ Hospital del Río Hortega, Valladolid, Spain

${ }^{3}$ Hospital Clínico Universitario, Santiago de Compostela, Spain.

AUTHOR'S ADDRESS: Roberto Hornero

E.T.S. Ingenieros de Telecomunicación

University of Valladolid

Camino del Cementerio s/ $\mathrm{n}^{\circ}$

47011 - Valladolid (Spain)

Phone: +34 983185570

Fax: +34 983423667 
Abstract- Approximate Entropy (ApEn) is a family of statistics introduced as a quantification of regularity in time series without any a priori knowledge about the system generating them. The aim of this preliminary study was to assess whether a time series analysis of arterial oxygen saturation $\left(\mathrm{SaO}_{2}\right)$ signals from overnight pulse oximetry by means of ApEn could yield essential information on the diagnosis of obstructive sleep apnea (OSA) syndrome. We analyzed $\mathrm{SaO}_{2}$ signals from 187 subjects: 111 with a positive diagnosis of OSA and 76 with a negative diagnosis of OSA. We divided our data in a training set (44 patients with OSA Positive and 30 patients with OSA Negative) and a test set (67 patients with OSA Positive and 46 patients with OSA Negative). The training set was used for algorithm development and optimum threshold selection. Results showed that recurrence of apnea events in patients with OSA determined a significant increase in ApEn values. This method was assessed prospectively using the test dataset, where we obtained 82.09\% sensitivity and $86.96 \%$ specificity. We conclude that ApEn analysis of arterial oxygen saturation from pulse oximetric recording could be useful in the study of OSA.

Index Terms - Apnea, approximate entropy, arterial oxygen saturation, overnight pulse oximetry, regularity, 


\section{INTRODUCTION}

Obstructive Sleep Apnea (OSA) syndrome is a respiratory disorder characterized by recurrent airflow obstruction caused by total or partial collapse on the upper airway [1]. Epidemiological studies in middle-aged populations have shown an OSA prevalence of $4 \%$ in males and $2 \%$ in females [2]. OSA has major health implications ranging from traffic accident to serious cardiac arrhythmias [3]. OSA is associated with increased risks of hypertension, myocardial infarction, and with increased mortality rates [4], [5].

At present, the most widely accepted diagnostic test for OSA syndrome is overnight polysomnography (PSG) [6]. PSG consists in the measurement and recording of several signals used to analyze sleep and breathing. The expensive and time-consuming nature of PSG prompts many sleep centers to perform an initial screening test in order to reduce the number of PSGs needed. Therefore, several strategies have been developed to reduce the number of sleep recordings, including sleep questionnaires, ambulatory recordings and simplified multi-channel systems. In sleep medicine, pulse oximetry is an essential tool for tracking the fluctuation in the arterial oxygen saturation $\left(\mathrm{SaO}_{2}\right)$ signals, which are characteristics for unstable ventilation in patients with sleep apnea. Pulse oximetry has provided early insights into sleep related breathing disturbances [7], and it has opened the way for subsequent systematic investigations of sleep apnea [8]. Thus, overnight pulse oximetry has been proposed as a simpler alternative to PSG in the diagnosis of OSA because it is readily available, it is relatively inexpensive and could potentially meet the large demand for diagnostic testing [9-12]. It can be easily done at home and repeated, which is not the case with PSG.

There are many quantitative indexes derived from overnight pulse oximetry in the diagnosis of 
OSA. The most widely used include the number of oxyhemoglobin desaturations below a certain threshold, usually 3 or $4 \%$ decline from baseline [13-16], or the cumulative time spent below a $\mathrm{SaO}_{2}$ of $90 \%[17,18]$. In this study, we estimated the entropy of $\mathrm{SaO}_{2}$ to predict the OSA syndrome.

Entropy is a concept addressing randomness and predictability, with greater entropy often associated with more randomness and less system order. Kolmogorov-Sinai entropy (K-S entropy), developed by Kolmogorov and expanded upon by Sinai, allows classifying deterministic dynamical systems by rate of information generation [19]. Unfortunately, K-S entropy was not developed for statistical applications and diverges to a value of infinity when the signal is contaminated by the slightest noise. A practical solution to this problem has been put forward using a recently developed family of statistics named Approximate Entropy (ApEn) [20]. Although there are many other entropy estimators, several properties of ApEn facilitate its utility for empirical time series analysis: it is nearly unaffected by noise below a de facto specified filter level; it can be applied to short time series and it is finite for stochastic, noisy deterministic and composite processes [21].

The goal of this study was to assess whether a time series analysis of arterial oxygen saturation $\left(\mathrm{SaO}_{2}\right)$ signals by means of $A p E n$ could yield essential information on the diagnosis of OSA syndrome.

\section{SUBJECTS}

One hundred and eighty seven subjects took part in this prospective study. Patients were recruited from the outpatient clinic. There were 147 males $(78.61 \%)$ and 40 females $(21.39 \%)$ with a mean \pm standard deviation (SD) age of $57.97 \pm 12.84$ years and mean body mass index 
(BMI) of $29.54 \pm 5.51$. All subjects were suspected of having OSA because of daytime hypersomnolence, loud snoring, nocturnal choking and awakenings, or apneic events (or all four symptoms) reported by the subject or a bedmate.

Sleep studies were carried out usually from midnight to 08:00 AM in the Sleep Unit of Hospital Clínico Universitario in Santiago de Compostela, Spain. The Review Board on Human Studies at this institution approved the protocol, and all subjects gave their informed consent to participate in the study. Patients were evaluated by an overnight pulse oximetry test in conjunction with a simultaneous conventional polysomnographic (PSG) study. This technique consisted in continuous monitoring using a polygraph (Ultrasom Network, Nicolet, Madison, WI, U.S.A.) and included EEG, electro-oculogram, chin electromyogram, air flow (three-port thermistor), ECG and measurement of chest wall movement. The PSG register was analyzed over periods of $30 \mathrm{~s}$ and during sleep phases I, II, III, IV and rapid eye movement, according to the system of Rechtschaffen and Kales [22]. Apnea was defined as the absence of air flow for more than $10 \mathrm{~s}$, and hypopnea as a decrease in respiratory flow of at least $50 \%$, accompanied by a decrease of more than $4 \%$ in the saturation of hemoglobin. The average apnea-hypopnea index (AHI) was calculated for hourly periods of sleep. In this study, an AHI $\geq 10$ events per hour was considered as diagnostic of OSA. If the subject had less than 3 hours of total sleep, the sleep study was repeated.

Recording of the arterial oxygen saturation $\left(\mathrm{SaO}_{2}\right)$ was carried out using a Criticare 504 oximeter (CSI, Waukeska, WI, U.S.A.) with a finger probe, with sampling frequency of $0.2 \mathrm{~Hz}$ (one sample every $5 \mathrm{~s}$ ). The $\mathrm{SaO}_{2}$ signals were saved to separate files and processed off-line by means of ApEn.

A positive diagnosis of OSA was confirmed in 111 (59.36\%) out of 187 subjects using PSG 
recordings. Table I summarizes the demographic data and clinical features for all subjects under study divided into patients with a positive diagnosis of OSA (OSA Positive group) and patients with a negative diagnosis of OSA (OSA Negative group). There were 94 males (84.68\%) and 17 females $(15.32 \%)$ in the OSA Positive group, with a mean \pm SD age of $58.30 \pm 12.88$ years and mean body mass index (BMI) of $30.45 \pm 4.92 \mathrm{~kg} / \mathrm{m}^{2}$. The mean age in the OSA Negative group was $57.57 \pm 12.87$ with a mean BMI of $28.42 \pm 6.02 \mathrm{~kg} / \mathrm{m}^{2}$. In this OSA negative group, there were 53 males (69.74\%) and 23 females (30.26\%) There were no significant differences between OSA Positive and Negative groups in mean age, mean body mass index and time recordings. However, the OSA Positive group presented a significant increase in the number of males (84.68\% vs. $69.74 \%)$.

We divided our population randomly into a training set (74 subjects, 39.57\%) and a test set (113 subjects, 60.43\%). The training set was used to develop the algorithm and to select the optimum threshold for ApEn method. The final algorithm was then applied without further alteration to the data from the test set. In the training set, we included 74 subjects ( 44 patients with a positive diagnosis of OSA and 30 with a negative diagnosis of OSA) with a mean age of $58.25 \pm 12.14$ years. In the test set there were 113 subjects (67 patients with a positive diagnosis of OSA and 46 with a negative diagnosis of OSA) with a mean age of $57.91 \pm 13.39$ years. The demographic data and clinical features of training set and test are summarized in Table I. Fig.1 shows $\mathrm{SaO}_{2}$ data plots from nocturnal oximetry for an OSA negative subject, an apparent OSA positive subject and an uncertain OSA positive subject.

\section{METHODS}

Approximate Entropy (ApEn) is a family of statistics introduced as a quantification of 
regularity in the data without any a priori knowledge about the system generating them [23]. It was constructed by Pincus [20], motivated by applications to short and noisy data sets, along thematically similar lines to K-S entropy. However, the focus was different: to provide a widely applicable, statistically valid formula that will distinguish data sets by a measure of regularity [20]. The observation motivating $A p E n$ is that if joint probability measures for reconstructed dynamics that describe each of two systems are different, then their marginal probability distributions on a fixed partition, given by conditional probabilities, are likely different. We typically need orders of magnitude fewer points to accurately estimate these marginal probabilities than to accurately reconstruct the attractor measure defining the process [21].

Several properties of ApEn facilitate its utility for empirical biomedical time series analysis. $A p E n$ is nearly unaffected by noise below a de facto specified filter level ( $r$ ), it is scale invariant and model independent, it evaluates both dominant and subordinated patterns in data, and it discriminates series for which clear feature recognition is difficult [21]. Moreover, it can be applied to time series of 50 or more points with good reproducibility; it is finite for stochastic, noisy deterministic and composite processes; and increasing values of ApEn correspond to more irregularity in the time series [21].

Based on numerous studies, ApEn correlates with "occult" or subclinical changes often undetected by other more classical time series means, including both moments statistics, and spectral and correlation analyses [21]. Moreover, ApEn changes have often been seen to be predictive of subsequent clinical changes. This has facilitated the application of ApEn to numerous settings. Within biology and medicine, it has been used to discriminate atypical EEGs [24] and respiratory patterns [25] from normative counterparts. Furthermore, it has been applied to quantify the differences in apparent regularity between the heart rate interval time series of 
aborted SIDS and healthy infants [26] and to characterize postoperative ventricular dysfunction [27]. Preliminary evidence suggests that applied to EEGs may be predictive of epileptic seizure [28] and may be an effective tool to quantify the depth of anesthesia [29]. Moreover, it has been shown that ApEn follows closely the results obtained from spectral entropy extracting features from EEG and respiratory recordings of a patient during Cheyne-Stokes respiration, with apparently higher sensitivity and reduced error [30]. Within endocrinology, it has been used in multifaceted ways; for instance, in the analysis of endocrine hormone release pulsatility [31] and the impact of pulsatility on the ensemble orderliness of neurohormone secretion [32].

ApEn assigns a non-negative number to a time series, with larger values corresponding to more irregularity in the data. Two input parameters, a run length $m$ and a tolerance window $r$, must be specified to compute ApEn. Briefly, ApEn measures the logarithmic likelihood that runs of patterns that are close (within $r$ ) for $m$ contiguous observations remain close (within the same tolerance width $r$ ) on subsequent incremental comparisons. It is important to consider $\operatorname{ApEn}(m, r)$ - or $\operatorname{ApEn}(m, r, N)$, where $N$ is the number of points of the time series - as a family of characterizing measures: comparisons between time series can only be made with the same values of $m$ and $r$ [21].

Formally, given $N$ data points from a time series $\{x(n)\}=x(1), x(2), \ldots, x(N)$, one should follow these steps to compute ApEn [21]:

1. Form $N-m+1$ vectors $X(1) \ldots X(N-m+1)$ defined by: $X(i)=[x(i), x(i+1), \ldots, x(i+m-1)], i=$ $1 \ldots N-m+1$. Each $i$-vector represents $m$ consecutive $x$ values, commencing with the $i$ th data point.

2. Define the distance between $X(i)$ and $X(j), d[X(i), X(j)]$, as the maximum absolute difference between their respective scalar components, i.e., the maximum norm: 


$$
d[X(i), X(j)]=\max _{k=1,2, \ldots, m}|x(i+k-1)-x(j+k-1)|
$$

3. For a given $X(i)$, count the number of $j(j=1 \ldots N-m+1, j \neq i)$ so that $d[X(i), X(j)] \leq r$, denoted as $N^{m}(i)$. Then, for $i=1 \ldots N-m+1$,

$$
C_{r}^{n}(i)=N^{m}(i) /(N-m+1)
$$

$C_{r}^{m}(i)$ measures, within a tolerance $r$, the frequency of patterns similar to a given one of window length $m$.

4. Compute the natural logarithm of each $C_{r}^{m}(i)$, and average it over $i$,

$$
\phi^{m}(r)=\frac{1}{N-m+1} \sum_{i=1}^{N-m+1} \ln C_{r}^{m}(i)
$$

5. Increase the dimension to $m+1$. Repeat steps 1) to 4) and find $C_{r}^{m+1}(i)$ and $\phi^{m+1}(r)$.

6. We define Approximate Entropy by:

$$
\operatorname{ApEn}(m, r, N)=\phi^{m}(r)-\phi^{m+1}(r)
$$

The accuracy and confidence of the entropy estimate improve as the number of matches of length $m$ and $m+1$ increase. The number of matches can be increased by choosing small $m$ 
(short templates) and large $r$ (wide tolerance). However, there are penalties for criteria that are too relaxed [20]. For small $r$ values, one usually achieves poor conditional probability estimates, while for large $r$ values, too much detailed system information is lost. To avoid a significant contribution of noise in an ApEn calculation, one must choose $r$ larger than most of the noise [20].

In this study, $\mathrm{SaO}_{2}$ signals were recorded with a sampling frequency of $0.2 \mathrm{~Hz}$ (one sample every 5 seconds). Then, the $\mathrm{SaO}_{2}$ signals were divided into epochs of 200 samples. Thus, each epoch to be analyzed by means of ApEn had a duration of 1000 seconds. As suggested by Pincus [21], ApEn was estimated using the widely established parameter values of $m=1, m=2$ and $r=$ $0.1,0.15,0.2$ and 0.25 times the standard deviation (SD) of the original data sequence $\{x(n)\}$. Normalizing $r$ in this manner gives ApEn a translation and scale invariance, in that it remains unchanged under uniform process magnification, reduction, or constant shift to higher or lower values [21]. These input parameters produce good statistical reproducibility for ApEn with time series of length $N \geq 60$, as considered herein [20], [23], [26], [33], [34].

\section{RESULTS}

We present the results obtained on the training and test sets.

\section{A. Training set}

$\operatorname{ApEn}(m, r, N)$ has to be seen as a family of characterizing measures, where $N$ is the number of points in our time series. As suggested by Pincus [21], we used $m=1$ and $m=2$ and four different values of $r: 0.1,0.15,0.2$ and 0.25 times the standard deviation (SD) of data. The results were averaged based on all the 200 sample epochs. Table II shows the mean ApEn values calculated from the $\mathrm{SaO}_{2}$ signal in the training set with different values of $m$ and $r$ to compare 
$\mathrm{SaO}_{2}$ signals from subjects in OSA Positive and Negative groups. We obtained significant differences $(p<0.01)$ with the Student's $t$-test with all the chosen values of $m$ and $r$. The best results (lowest $p$-value) were obtained with $m=1$ and $r=0.25$ times the SD of the data. Larger values of ApEn imply substantial fluctuation, or irregularity, in the time series [21]. Thus, the smaller ApEn values in the $\mathrm{SaO}_{2}$ signals from OSA Negative group imply stronger regularity, or persistence, than in the $\mathrm{SaO}_{2}$ signals from OSA Positive group.

We selected the optimum threshold from the training set to improve the sensitivity/specificity pair according to the receiver operating characteristic (ROC) plots [35]. We used a computer program developed with Matlab ${ }^{\circledR}$ that automatically selected different thresholds or cut-off points (ApEn values) and calculated the sensitivity/specificity pair for each one of them. Sensitivity the true positive rate - is the proportion of patients with a positive diagnosis of OSA who test positive (ApEn value higher than the cut-off point), whereas specificity - the true negative rate represents the percentage of patients with a negative diagnosis of OSA correctly recognized (ApEn value lower than the cut-off point). The program selected the optimal threshold as the cutoff point determined graphically from the ROC curve as the point closest to the left top point (100\% sensitivity, $100 \%$ specificity).

Fig. 2 shows this ROC curve from the training set for $A p E n(m=1, r=0.25 \mathrm{SD})$. We obtained an optimum threshold $l=0.77$ and an area under the ROC curve of 0.94 , close to the ideal setting of a perfect diagnostic test (area under the ROC curve of 1). Moreover, we achieved $81.82 \%$ sensitivity and $90 \%$ specificity in the training set with this optimum threshold $(l=0.77)$.

Therefore, we selected $m=1$ and $r=0.25$ times the SD of the original data sequence in the ApEn estimation from $\mathrm{SaO}_{2}$ signals. ApEn values for OSA Positive and OSA Negative groups in the training set are shown in Fig. 3 with the optimum threshold at $l=0.77$. It can be seen that 
patients in OSA Negative group had lower ApEn values $(0.46 \pm 0.23)$ than patients in OSA Positive group $(1.01 \pm 0.26)$.

\section{B. Test set}

We used the parameters chosen from the study of the training set time series: $m=1$ and $r=$ 0.25 times the $\mathrm{SD}$ of the time series in the ApEn estimation of $\mathrm{SaO}_{2}$ signals from the test set. Fig. 4 represents the mean ApEn values for OSA Positive and OSA Negative groups in the test set. ApEn values were higher in the OSA Positive group $(1.04 \pm 0.29)$ than in the OSA Negative group $(0.48 \pm 0.26)$. These results confirmed that the degree of irregularity of $\mathrm{SaO}_{2}$ signals was higher in the OSA Positive group. Applying the $t$-Student test, we verified that there was a significant difference $(p<0.01)$.

Moreover, we computed sensitivity and specificity in the test set with the previously selected threshold. We achieved $82.09 \%$ sensitivity and $86.96 \%$ specificity with this test set.

\section{DisCUSSION AND CONCLUSIONS}

In this study, we analyzed the arterial oxygen saturation $(\mathrm{SaO} 2)$ signals of 187 patients (111 with a positive diagnosis of OSA and 76 with a negative diagnosis of OSA) by means of ApEn, a non-linear method that quantifies the regularity of time series. To avoid the optimization of all parameters involved (method/measure parameters and threshold) on the whole data record, we divided our data into a training set (44 patients with OSA Positive and 30 patients with OSA Negative) and a test set (67 patients with OSA Positive and 46 patients with OSA Negative). The training set was used for algorithm development and optimum threshold selection. The final algorithm was then applied to the test set, where we obtained $82.09 \%$ sensitivity and $86.96 \%$ specificity. 
Results showed that the recurrence of apnea events in patients with OSA determined a significant increase in the $A p E n$ values of $\mathrm{SaO}_{2}$ signals. $A p E n(m=1, r=0.25 \mathrm{SD})$ reflects the rate of new pattern generation when the dimension $m$ decreases from 2 to 1 [36]. A larger value of ApEn means that the chance of new pattern generation is greater, so the sequence is more irregular or complex, and vice versa. In OSA, oxygen desaturations associated with apnea events cause fluctuations in $\mathrm{SaO}_{2}$ signal leading to high $A p E n$ values.

Several quantitative indexes estimated from overnight pulse oximetry have been used to predict the presence of OSA. These indexes include the number of oxyhemoglobin desaturations below a certain threshold, usually 3 or $4 \%$ decline from baseline [13-16], the cumulative time spent below a $\mathrm{SaO}_{2}$ of $90 \%[17,18]$, saturation impairment time index that integrates severity and duration of oxygen desaturation [37], spectral analysis of $\mathrm{SaO}_{2}$ and pulse rate applied to evaluate the presence or absence of a peak in the periodogram [38], or a measure of the variability of the $\mathrm{SaO}_{2}(\Delta$ index $)[8,18]$. In these studies, the sensitivity of pulse oximetry ranged from 88 to $98 \%$, and the specificity from 40 to $88 \%$ if an apnea-hipopnea index (AHI) of 10 or 15 events per hour measured during polysomnography was defined as the upper limit of normalcy. However, there are some methodological differences that do not allow comparing all these results with our study. At present, there is not a definite standard AHI criterion for the diagnosis of OSA with the threshold varying from 5 to 20 events per hour. Thus, there is not an agreement on the standard threshold to diagnose OSA [18]. Some works use conservative values and employ an AHI $\geq 15$ events per hour to determine OSA, whereas other researchers diagnose mild OSA with an AHI $\geq 5$. The study by Vazquez et al. [13] reported the highest sensitivity (98\%) and specificity (88\%). However, they used a definition of arousal that differs substantially from the criteria proposed by the Atlas Task Force [39]. As a result of their definition of 
hypopnea, results will differ substantially from estimated in conventional studies, as it is indicated in [18]. Other works [16-18, 37-38] achieved better sensitivity values, ranging from 88 to $98 \%$, than our study, but failed in specificity (from 40 to $75 \%$ ). We used a training set to develop the algorithm and to select the optimum threshold (ApEn value) to improve the sensitivity/specificity pair according to ROC curve. With a test set, we obtained a higher specificity $(86.96 \%)$ but a lower sensitivity $(82.09 \%)$ than other studies. Moreover, when comparing our results with these previous studies on oximetry, we can confirm several aspects about reduction of variability in the oxygen saturation signals in non-OSA subjects $[8,18]$

Certain limitations that could influence our results need to be considered. Firstly, there are some technical problems associated with oximetry for recognizing OSA [38]. For example, there may be poor contact between the probe and the finger due to body movements, and bad regional circulation occasionally produces signals resembling multiple falls in oxygen saturation. Mindful of this, we examined our recordings before the analysis to see if they evidenced technical problems. For example, we eliminated all data that registered drops to zero. From our experience, we know these falls are generally due to finger-probe disconnections. Secondly, patients with positive OSA were predominantly studied. Therefore, the sample may not fully represent the population of patients referred to clinicians, and those having snoring or upper airway resistance. A third limitation to the applicability of our method should be mentioned. We used overnight pulse oximetry obtained simultaneously with PSG data in the sleep laboratory. This has the advantage of eliminating such potential confounders as night to night variability of AHI, as well as ensuring that oximetry data were collected in exactly the same environment as the PSG data. Further analyses using overnight oximetry done in the home settings unattended by technicians are necessary. 
In summary, we found that the recurrence of apnea events in patients with OSA determined a significant increase in the ApEn values of the arterial oxygen saturation $\left(\mathrm{SaO}_{2}\right)$ signals. This method was assessed prospectively using the test dataset, where we obtained $82.09 \%$ sensitivity and $86.96 \%$ specificity To our knowledge, this is the first study that uses ApEn to verify the diagnostic value of oximetry recordings in OSA syndrome. We think that ApEn analysis of nocturnal pulse oximetry recording could be a supplementary method to the conventional indexes for diagnosis of OSA on an ambulatory basis. However, further work is now required to test the potential value of our methodology with a larger data set. 
TABLE I

DEMOGRAPHIC AND CLINICAL FEATURES FOR ALL SUBJECTS UNDER STUDY, AND THE TRAINING AND TEST SETS

\begin{tabular}{|c|c|c|c|}
\hline \multicolumn{4}{|c|}{ ALL SUBJECTS } \\
\hline & All & OSA Positive & OSA Negative \\
\hline Subjects $(n)$ & 187 & 111 & 76 \\
\hline Age (years) & $57.97 \pm 12.84$ & $58.30 \pm 12.88$ & $57.57 \pm 12.87$ \\
\hline Males (\%) & 78.61 & 84.68 & 69.74 \\
\hline$B M I\left(k g / m^{2}\right)$ & $29.54 \pm 5.51$ & $30.45 \pm 4.92$ & $28.42 \pm 6.02$ \\
\hline Recording Time $(h)$ & $8.19 \pm 0.62$ & $8.17 \pm 0.75$ & $8.22 \pm 0.33$ \\
\hline$A H I(n / h)$ & & $40.07 \pm 19.64$ & $2.04 \pm 2.36$ \\
\hline \multicolumn{4}{|c|}{ TRAINING SET } \\
\hline & All & OSA Positive & OSA Negative \\
\hline Subjects $(n)$ & 74 & 44 & 30 \\
\hline Age (years) & $58.25 \pm 12.14$ & $56.73 \pm 13.61$ & $59.59 \pm 10.19$ \\
\hline Males (\%) & 75.68 & 79.55 & 70.00 \\
\hline$B M I\left(k g / m^{2}\right)$ & $29.62 \pm 5.71$ & $30.19 \pm 5.09$ & $28.93 \pm 6.40$ \\
\hline Recording Time $(h)$ & $8.22 \pm 0.41$ & $8.20 \pm 0.49$ & $8.25 \pm 0.27$ \\
\hline$A H I(n / h)$ & & $38.11 \pm 18.18$ & $2.60 \pm 2.51$ \\
\hline \multicolumn{4}{|c|}{ TEST SET } \\
\hline & All & OSA Positive & OSA Negative \\
\hline Subjects $(n)$ & 113 & 67 & 46 \\
\hline Age (years) & $57.91 \pm 13.39$ & $59.37 \pm 12.38$ & $56.03 \pm 14.54$ \\
\hline Males (\%) & 80.53 & 88.06 & 69.57 \\
\hline$B M I\left(k g / m^{2}\right)$ & $29.49 \pm 5.41$ & $30.63 \pm 4.84$ & $28.07 \pm 5.80$ \\
\hline Recording Time(h) & $8.17 \pm 0.72$ & $8.14 \pm 0.88$ & $8.20 \pm 0.37$ \\
\hline$A H I(n / h)$ & & $41.36 \pm 20.58$ & $1.67 \pm 2.21$ \\
\hline
\end{tabular}

Data are presented as mean \pm SD or $n(\%)$. OSA Positive: patients with a positive diagnosis of obstructive sleep apnea syndrome; OSA Negative: subjects with a negative diagnosis of obstructive sleep apnea syndrome; BMI: body mass index; AHI $(n / h)$ : apnea/hypopnea index calculated for hourly periods. 


\section{TABLE II}

AVERAGE $A p E n(m, r)$ VALUES ESTIMATED FROM OXYGEN SATURATION $\left(\mathrm{SAO}_{2}\right)$ SIGNALS OF SUBJECTS IN THE TRAINING SET USING $m=1$ AND $m=2$, AND $r=0.1,0.15,0.2,0.25$ TIMES THE STANDARD DEVIATION (SD) OF THE ORIGINAL DATA SEQUENCE

\begin{tabular}{ccccc}
\hline \hline $\boldsymbol{m}$ & $\boldsymbol{r}$ & $\begin{array}{c}\boldsymbol{A p E n}(\boldsymbol{m}, \boldsymbol{r}) \\
\text { OSA POSITIVE }\end{array}$ & $\begin{array}{c}\text { ApEn(m,r) } \\
\text { OSA NEGATIVE }\end{array}$ & $\begin{array}{c}\text { t-test } \\
\boldsymbol{p} \text {-value }\end{array}$ \\
\hline 1 & 0.1 SD & $1.13 \pm 0.34$ & $0.48 \pm 0.27$ & $p<0.01$ \\
1 & $0.15 \mathrm{SD}$ & $1.11 \pm 0.32$ & $0.48 \pm 0.25$ & $p<0.01$ \\
1 & 0.2 SD & $1.07 \pm 0.30$ & $0.47 \pm 0.25$ & $p<0.01$ \\
1 & $0.25 \mathrm{SD}$ & $1.01 \pm 0.26$ & $0.46 \pm 0.23$ & $p<0.01$ \\
2 & $0.1 \mathrm{SD}$ & $0.62 \pm 0.09$ & $0.39 \pm 0.17$ & $p<0.01$ \\
2 & $0.15 \mathrm{SD}$ & $0.64 \pm 0.09$ & $0.39 \pm 0.17$ & $p<0.01$ \\
2 & $0.2 \mathrm{SD}$ & $0.66 \pm 0.09$ & $0.39 \pm 0.17$ & $p<0.01$ \\
2 & $0.25 \mathrm{SD}$ & $0.67 \pm 0.11$ & $0.39 \pm 0.17$ & $p<0.01$ \\
\hline \hline
\end{tabular}




\section{FIGURE LEGENDS}

Fig. 1. $\mathrm{SaO}_{2}$ records from nocturnal oximetry for (a) a common OSA negative subject, (b) an apparent OSA positive patient and (c) an uncertain OSA positive subject.

Fig. 2. ROC curve for the $A p E n(m=1, r=0.25 \mathrm{SD})$ values from oxygen saturation $(\mathrm{SaO} 2)$ signal in the diagnosis of the obstructive sleep apnea syndrome in the training set. We selected different thresholds or cut-off points (ApEn values) and calculated the sensitivity/specificity pair for each one of them. The symbol $\downarrow$ indicates the optimum threshold.

Fig. 3. Mean ApEn values of oxygen saturation $\left(\mathrm{SaO}_{2}\right)$ signal for patients in the OSA Positive group and OSA Negative group in the training set. The optimum threshold, set at 0.77 , is represented with a dashed line.

Fig. 4. Mean ApEn values of oxygen saturation $\left(\mathrm{SaO}_{2}\right)$ signal for patients in the OSA Positive group and OSA Negative group in the test set. The optimum threshold, set at 0.77 , is represented with a dashed line. 


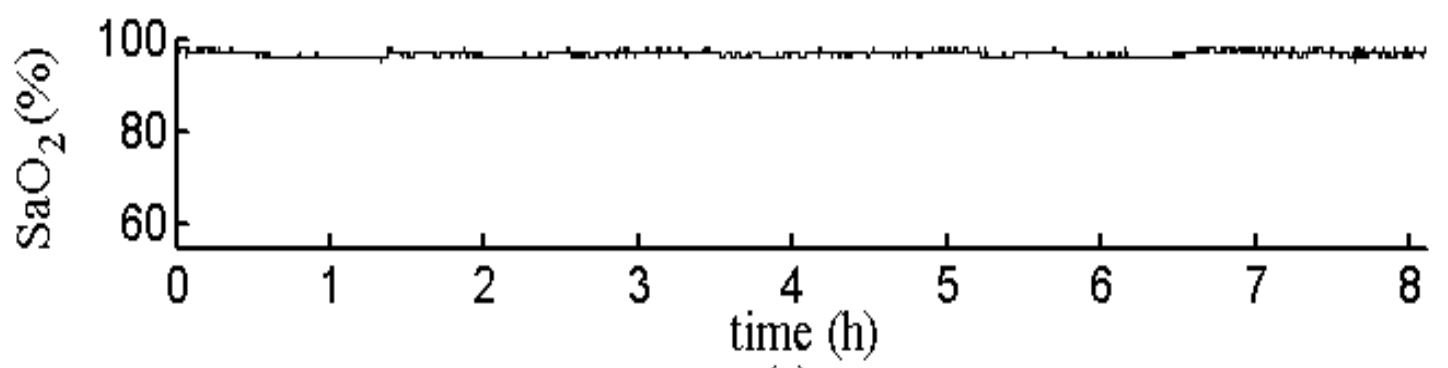

(a)

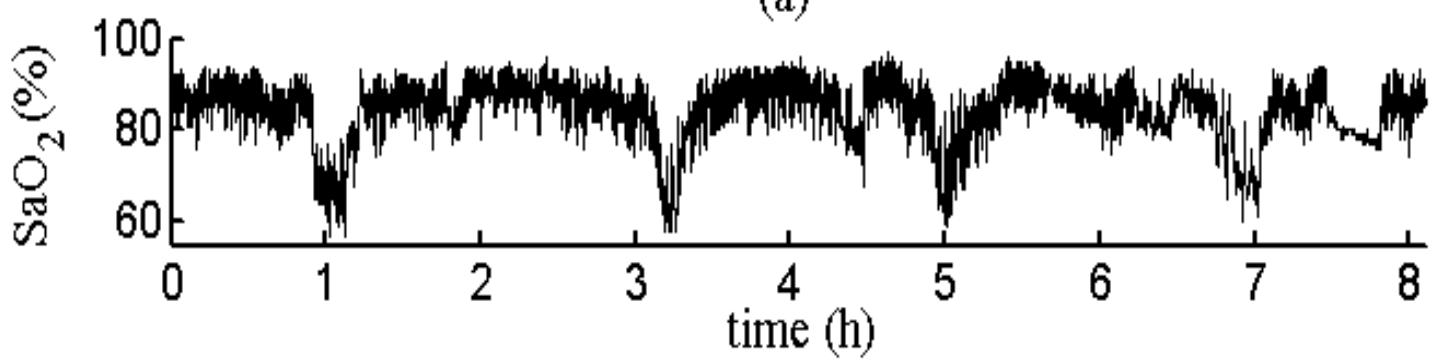

(b)

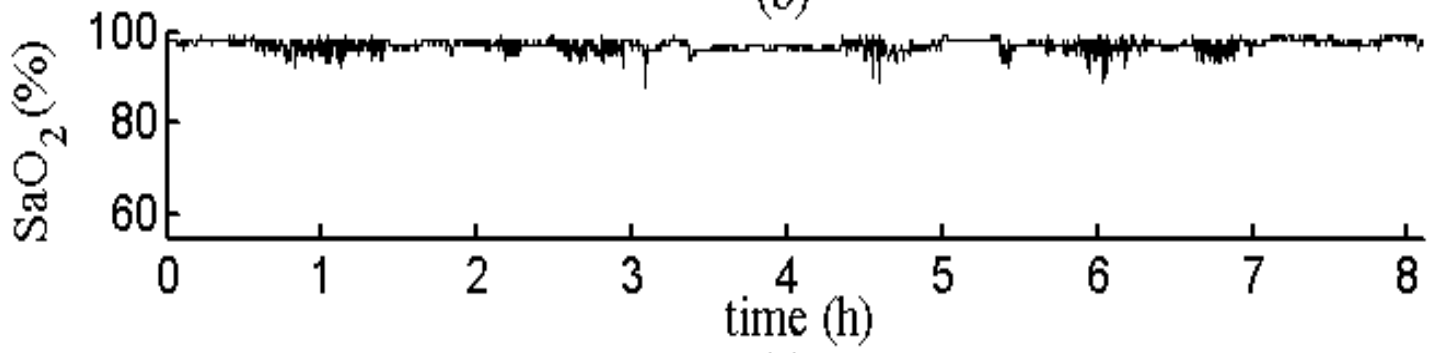

(c)

Fig. 1. $\mathrm{SaO}_{2}$ records from nocturnal oximetry for (a) a common OSA negative subject, (b) an apparent OSA positive patient and (c) an uncertain OSA positive subject. 


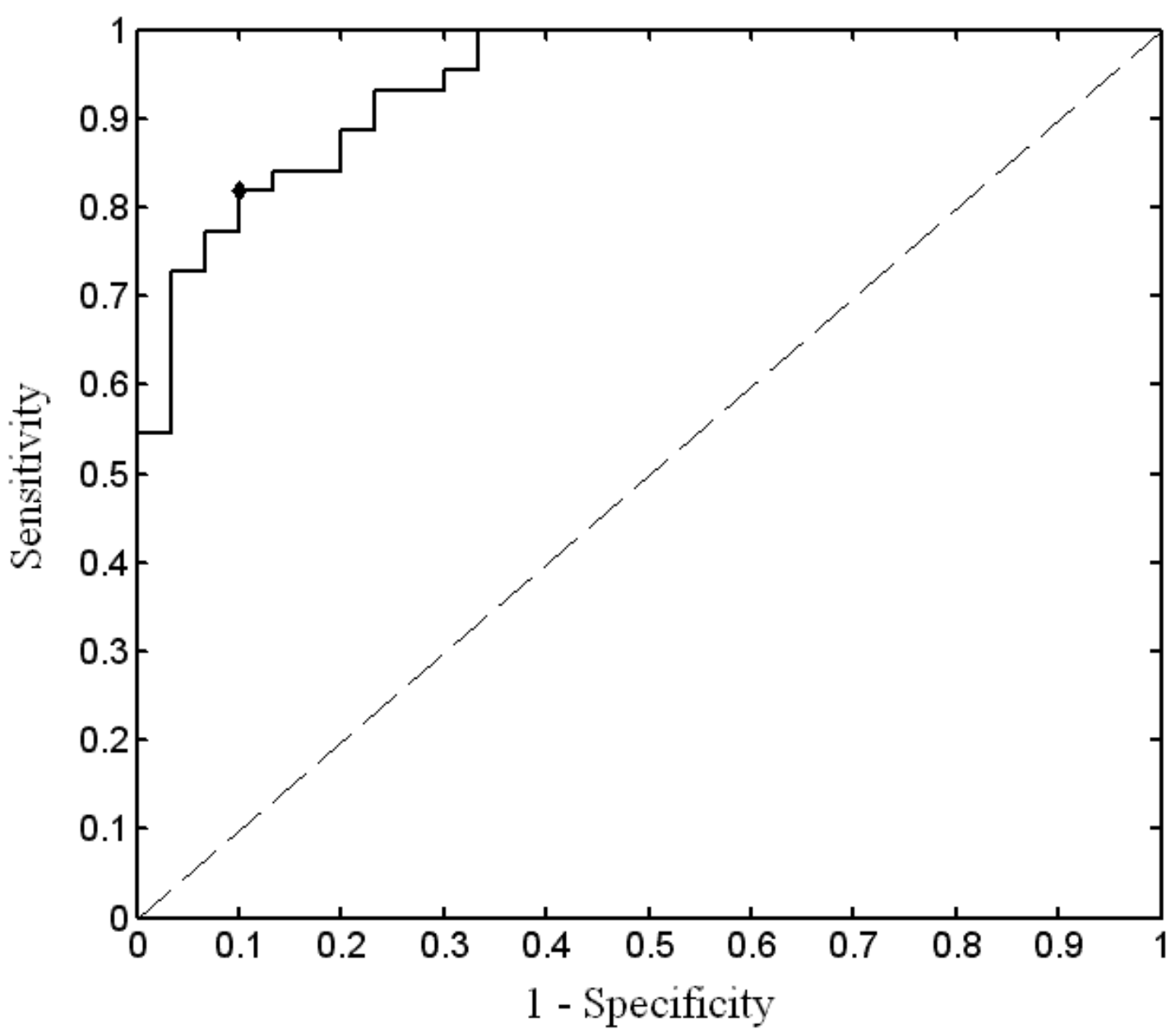

Fig. 2. ROC curve for the $A p E n(m=1, r=0.25 \mathrm{SD})$ values from oxygen saturation $(\mathrm{SaO} 2)$ signal in the diagnosis of the obstructive sleep apnea syndrome in the training set. We selected different thresholds or cut-off points (ApEn values) and calculated the sensitivity/specificity pair for each one of them. The symbol $\downarrow$ indicates the optimum threshold. 


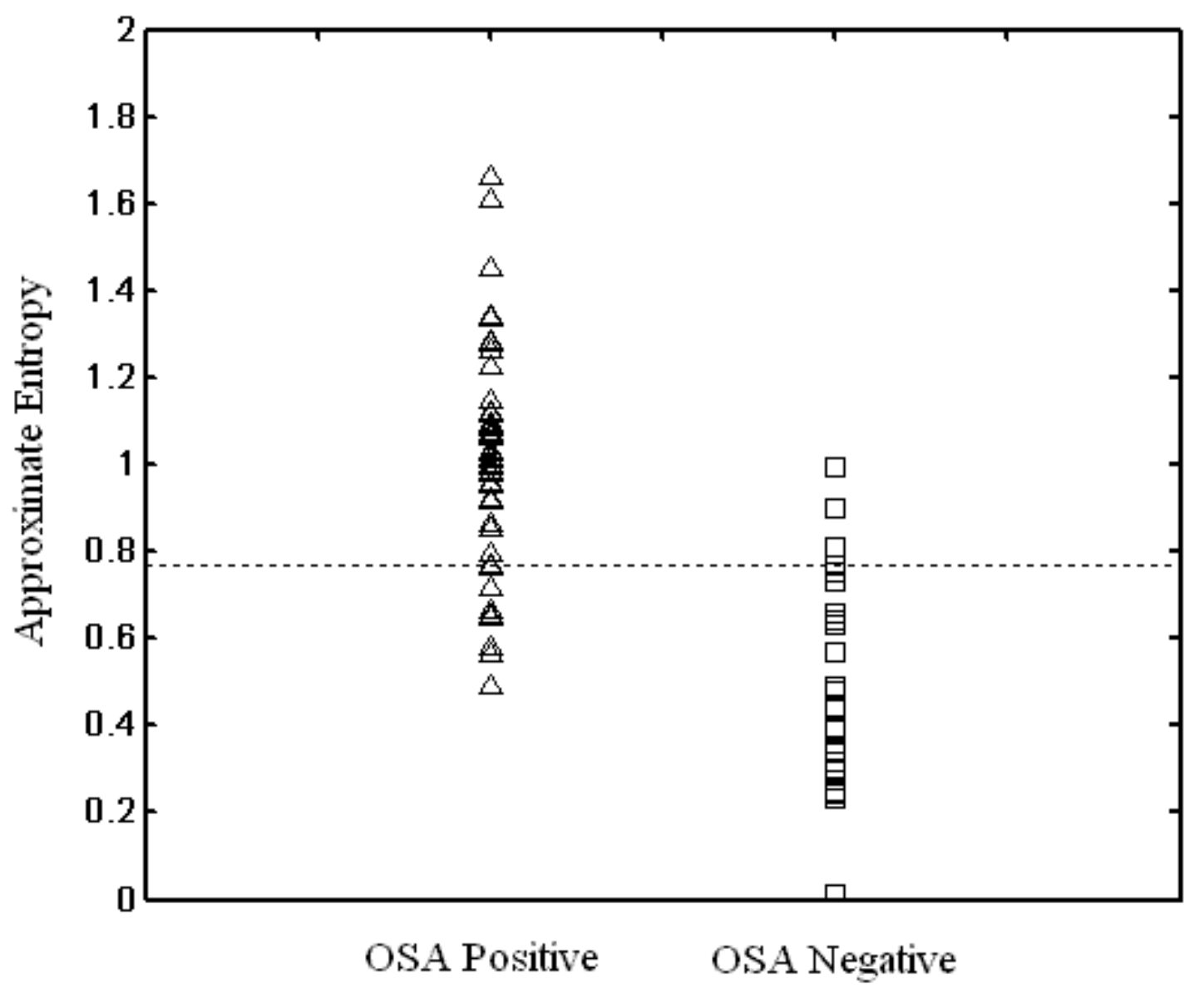

Fig. 3. Mean ApEn values of oxygen saturation $\left(\mathrm{SaO}_{2}\right)$ signal for patients in the OSA Positive group and OSA Negative group in the training set. The optimum threshold, set at 0.77 , is represented with a dashed line. 


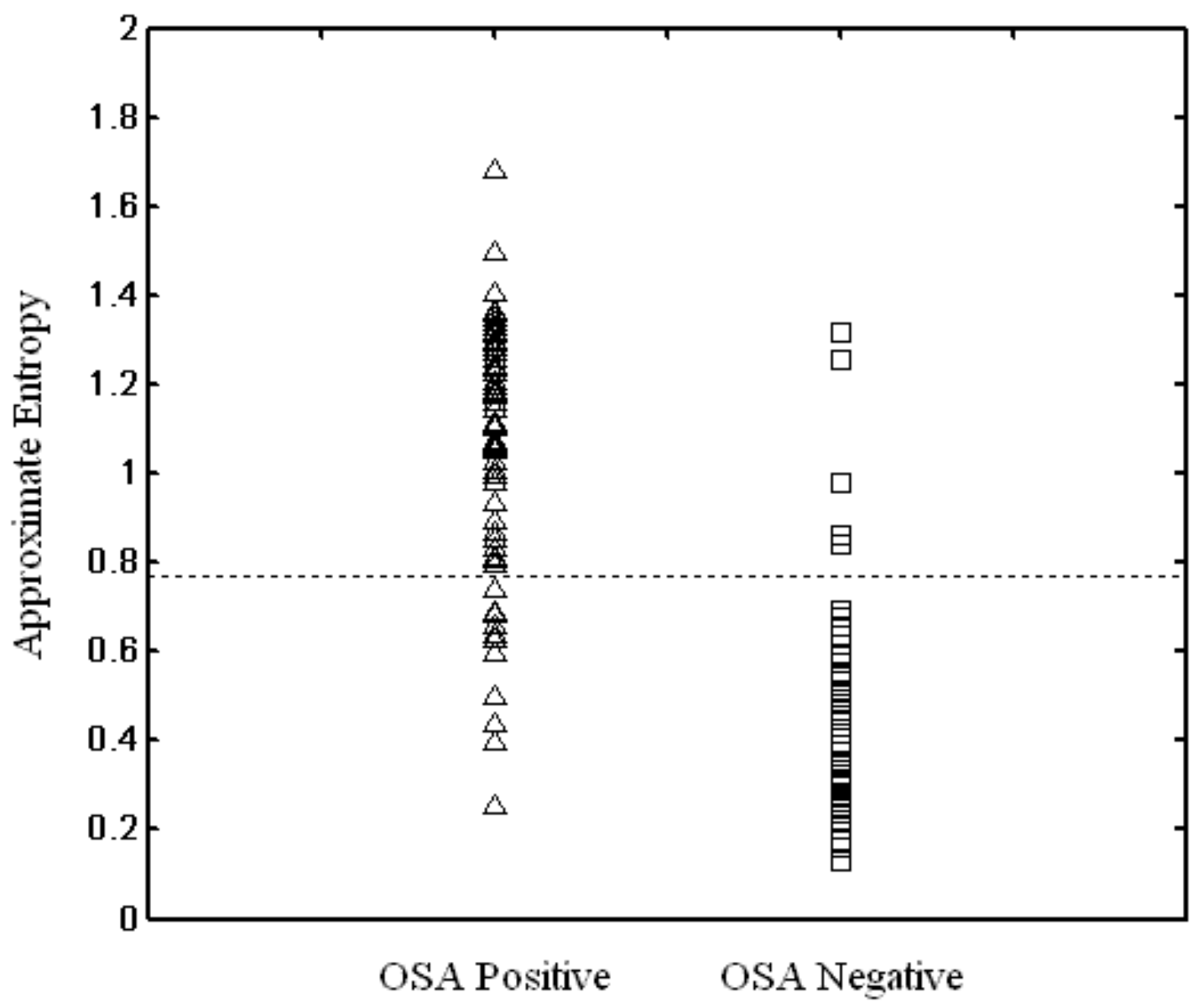

Fig. 4. Mean ApEn values of oxygen saturation $\left(\mathrm{SaO}_{2}\right)$ signal for patients in the OSA Positive group and OSA Negative group in the test set. The optimum threshold, set at 0.77 , is represented with a dashed line. 


\section{REFERENCES}

[1] J. E. Remmers, W. J. DeGroot, E. K. Saurland, and A. M. Anch, "Pathogenesis of upper airway occlusion during sleep,” J. Appl. Physiol., vol. 44, pp. 931-938, Jun. 1978.

[2] T. Young, M. Palta, J. Dempsey. S. Skatrud. S. Weber, and S. Badr, "The occurrence of sleep-disordered breathing among middle-aged adults," N. Engl. J. Med., vol. 328, pp. 12301235, Apr. 1993.

[3] F. Barbé, J. Pericas, A. Muñoz, L. Findley, J.M. Anto, and A. Agustí, “Automobile accidents in patients with sleep apnea syndrome. An epidemiological study," Am. J. Respir. Crit. Care Med., vol. 158, pp. 18-22, Jul. 1998.

[4] P. Lavie, R. Peled, I. Berger, N. Yoff, J. Zomer, and A. H. Rubin, "Mortality in sleep apnea patients: a multivariate analysis of risk factors," Sleep, vol. 18, pp. 149-157, Apr. 1995.

[5] J. He, M. H. Kryger, F. J. Zorick, W. Conway, and T. Roth, "Mortality and apnea index in obstructive sleep apnea: experience in 385 male patients," Chest, vol. 94, pp. 9-14, Jul. 1988.

[6] Agency for Health Care Policy and Research (AHCPR), "Systematic review of the literature regarding the diagnosis of sleep apnea: summary, evidence report/technology assessment number 1," Department of Health and Human Services, U.S. Public Health Service, vol. E002, Feb. 1999.

[7] A. J. Block, P. G. Boysen, J. W. Wynne, and L. A. Hunt, "Sleep apnea, hypopnea and oxygen desaturation in normal subjects," N. Engl. J. Med., vol. 300, pp. 513-517, Mar. 1979.

[8] A. J. Block, "Getting the most out of nocturnal pulse oximetry," Chest, vol. 125, pp. 16281630, Nov. 2003. 
[9] F. Series, I. Marc, and Y. Cormier, "Utility of nocturnal home oximetry for case finding in patients with suspected sleep apnea hypopnea syndrome," Ann. Intern. Med., vol. 119, pp. 449-453, Sep. 1993.

[10] P. Levy, J. L. Pepin, C. Deschaux-Blanc, B. Paramelle, and C. Brambilla, "Accuracy of oximetry for detection of respiratory disturbances in sleep apnea syndrome," Chest, vol. 109, pp. 395-399, Feb. 1996.

[11]L. J. Epstein and G. R. Dorlac, "Cost-effectiveness analysis of nocturnal oximetry as a method of screening for sleep apnea-hypopnea syndrome," Chest, vol 113, pp. 97-103, Jan. 1998.

[12]E. Chiner, J. Signes-Costa, J. M. Arriero, J. Marco, I. Fuentes and A. Sergado, “A Nocturnal oximetry for the diagnosis of the sleep apnea hypopnea syndrome: a method to reduce the number of polysomnographies?,” Thorax, vol. 54, pp. 968-971, Nov. 1999.

[13] J. Vazquez, W. H. Tsai, W. W. Flemons, A. Masuda, R. Brant, E. Hajduk, W. A. Whitelaw, and J. E. Remmers, "Automated analysis of digital oximetry in the diagnosis of obstructive sleep apnea”, Thorax, vol. 55, pp. 302-307, Apr. 2000.

[14]B. H. Taha, J. A. Dempsey, and S. M. Weber, "Automated detection and classification of sleep-disorders breathing from conventional polysomnography data," Sleep, vol. 20, pp. 9911001, Nov. 1997.

[15]H. Rauscher, W. Popp, and H. Zwick, “Computerized detection of respiratory events during sleep from rapid increase in oxyhemoglobin saturation," Lung, vol. 169, pp. 335-342, 1991.

[16] Y. Yamashiro and M. H. Kryger, "Nocturnal oximetry: is it a screening tool for sleep disorders?," Sleep, vol. 18, pp. 167-171, Apr. 1995. 
[17] S. Gyulay, L. G. Olson, M. J. Hensley, M. T. King, K. M. Allen and N. A. Saunders, “A comparison of clinical assessment and home oximetry in the diagnosis of obstructive sleep apnea," Am. Rev. Respir. Di., vol 147, pp. 50-53, Jan. 1993.

[18] U. J. Magalang, J. Dmochowski, S. Veeramachaneni, A. Draw, M. J. Mador, A. El-Solh and B. J. Grant, "Prediction of the apnea-hypopnea index from overnight pulse oximetry," Chest, vol. 124, pp. 1694-1701, Nov. 2003.

[19]A. N. Kolmogorov, “A new metric invariant of transient dynamical systems and automorphisms in Lebesgue spaces," Dokl Akad Nauk SSSR, vol. 119, pp.861-864, 1958.

[20] S. M. Pincus, "Approximate entropy as a measure of system complexity," Proc. Natl. Acad. Sci. USA, vol. 88, pp. 2297-2301, Mar. 1991.

[21] S. M. Pincus, "Assessing serial irregularity and its implications for health," Ann. NY Acad. Sci., vol. 954, pp. 245-267, Dec. 2001.

[22] A. Rechtschaffen and A. Kales, "A Manual of Standardized Terminology, Techniques and Scoring System for Sleep Stages of Human Subjects,” Brain Information Services, Brain Research Institute, University of California, Los Angeles , 1968.

[23] S. M. Pincus and A. L. Goldberger, "Physiological time series analysis: what does regularity quantify?," Am. J. Physiol. (Heart Circ. Physiol.), vol. 266, pp. H1643-H1656, Apr. 1994.

[24] J. Bruhn, H. Röpcke, B. Rehberg, T. Bouillon, and A. Hoeft, "Electroencephalogram approximate entropy correctly classifies the occurrence of burst suppression pattern as increasing anesthetic drug effect,” Anesthesiology, vol. 93, pp. 981-985, Oct. 2000.

[25] M. Engoren, "Approximate entropy of respiratory rate and tidal volume during weaning from mechanical ventillation," Crit. Care Med., vol. 26, pp. 1817-1823, Nov. 1998. 
[26] S. M. Pincus, T. R. Cummings, and G. G. Haddad, "Heart rate control in normal and aborted SIDS infants," Am. J. Physiol. (Regulatory Integrative Comp. Physiol.), vol. 264, pp. R638R646, Mar. 1993.

[27]L. A. Fleisher, S. M. Pincus, and S. H. Rosenbaum, “Approximate entropy of heart rate as a correlate of postoperative ventricular dysfunction," Anesthesiology, vol. 78, pp. 683-692, 1993.

[28] N. Radhakrishnan and B. N. Gangadhar, "Estimating regularity in epileptic seizure timeseries data. A complexity-measure approach," IEEE Eng. Med. Biol. Mag., vol. 17, pp. 8994, May-Jun. 1998.

[29] X.-S. Zhang and R. J. Roy, "Derived fuzzy knowledge model for estimating the depth of anesthesia," IEEE Trans. Biomed. Eng., vol. 48, pp. 312-323, Mar. 2001.

[30] I. A. Rezek and S. J. Roberts, "Stochastic Complexity Measures for Physiological Signal Analysis," IEEE Trans. Biomed. Eng., vol. 45, pp. 1186-1191, Sep. 1998.

[31]S. M. Pincus, "Older males secrete luteinizing hormone and testosterone more irregularly and joint more asynchronously, than younger males," Proc. Natl. Acad. Sci. USA, vol. 93, pp. 14100-14105, Nov. 1996.

[32] J. D. Veldhuis, M. L. Johnson, O. L. Veldhuis, M. Straume, and S. M. Pincus, "Impact of pulsatility on the ensemble orderliness (approximate entropy) of neurohormone secretion," Am. J. Physiol. (Regulatory Integrative Comp. Physiol.), vol. 281, pp. 1975-1985, Dec. 2001.

[33] S. M. Pincus and D. L. Keefe, "Quantification of hormone pulsatility via an approximate entropy algorithm," Am. J. Physiol. (Endocrinol. Metab.), vol. 262, pp. E741-E754, May 1992. 
[34]D. T. Kaplan, M. I. Furman, S. M. Pincus, S. M. Ryan, L. A. Lipsitz, and A. L. Goldberger, “Aging and the complexity of cardiovascular dynamics," Byophys. J., vol. 59, pp. 945-949, Apr. 1991.

[35]M. H. Zweig and G. Campbell, "Receiver-Operating Characteristic (ROC) plots: a fundamental evaluation tool in clinical medicine," Clin. Chem., vol. 39, pp. 561-577, Apr. 1993.

[36] Y. Fusheng, H. Bo, and T. Qingyu, “Approximate entropy and its application in biosignal analysis". In Nonlinear Biomedical Signal Processing Volume II: Dynamic Analysis and Modeling, M. Akay. New York: IEEE Press Series on Biomedical Engineering, 2001, pp. 72 91

[37] A. L. Chesson, W.M. Anderson, R.C. Walls, and L.E. Bairnsfather, "Assessment of hypoxemia in patients with sleep disorders using saturation impairment time," Am. Rev. Respir. Dis., vol. 148, pp. 1592-1598, Dec. 1993.

[38] C. Zamarrón, P. V. Romero, J. R. Rodríguez, and F. Gude, “Oximetry spectral analysis in the diagnosis of obstructive sleep apnoea," Clinical Science, vol. 97, pp. 467-473, Oct. 1999.

[39] The Atlas Task Force, "EEG arousals: scoring rules and examples. A preliminary report from the Sleep Disorder Task Force of the American Sleep Disorders Association," Sleep, vol. 20, pp. 991-1001, Jun. 1997. 